\title{
Hepatitis B Vaccination: Needs a Revision
}

\author{
Mohammad Saeid Rezaee-Zavareh ${ }^{1,2}$; Behzad Einollahi ${ }^{3, *}$ \\ ${ }^{1}$ Students' Research Committee, Baqiyatallah University of Medical Sciences, Tehran, IR Iran \\ 2 2 Middle East Liver Disease Center, Tehran, IR Iran \\ ${ }^{3}$ Nephrology and Urology Research Center, Baqiyatallah University of Medical Sciences, Tehran, IR Iran \\ ${ }^{*}$ Corresponding Author: Behzad Einollahi, Nephrology and Urology Research Center, Baqiyatallah University of Medical Sciences, Tehran, IR Iran. Tel/Fax: +98-2181262073, E-mail: \\ einollahi@numonthly.com.ir \\ Received: January 8, 2014; Accepted: January 8, 2014
}

Keywords:Hepatitis B Antibodies; Hepatitis B Vaccines; Vaccination; Hepatitis B Surface Antigens

\section{Hepatitis B virus Infection, Epidemiology and Complications}

It is common knowledge that hepatitis B virus (HBV) infection is an important worldwide health issue which can lead to acute and chronic infection Fulminant hepatitis may be the result of the acute infection. HBV infection in chronic condition can also increase the risk of some more important complications such as hepatic decompensation, cirrhosis and even eventually hepatocellular carcinoma (HCC) $(1,2)$. Based on the world health organization (WHO) reports, there are more than two billion individuals infected with HBV infection around the world (3). Almost 350 million people have chronic liver disease and the mortality rate, as a consequence of hepatitis B is approximately one million each year as well as over 4 million new cases of HBV infections (3).

\section{History of HBV Vaccine and Its Generations}

Like for many other infectious diseases, vaccination, as a preventive method, plays an important role in disease control of the HBV infection (4). Until now, three generations of HBV vaccines has been introduced. Krugman's observation about immunogenicity of HBsAg and the protective effects of anti-HBS antibody against HBV finally led to generating the first vaccine generation (1) containing inactivated HBsAg driven from the human carrier plasma of the HBV infected individuals. This vaccine was produced by Merck and at the same time by the Pasteur institute. Subsequently, it was approved by the FDA in 1981 (1). Recombinant DNA technology let to making second generation of HBV vaccines by using the yeast Saccharomyces cerevisiae with these two formulas; Engerix B and Recombivax HB. These types of vaccination also contain HBsAg. However, third generation vaccines are considerably more immunogenic, than HBsAg due to their uses of pre-S1 and pre-S2 antigens, but they aren't used widespread yet. Third generation vaccines are also manufactured with recombinant DNA technology by mammalian cells $(1,5)$.

After the WHO suggestion in 1991 for application of a widespread HBV vaccination by 1997, many countries including the USA and some regions of East Asia and Europe saw a significant decrease in prevalence of the HBV infection $(5,6)$, childhood HCC and fulminant hepatitis. (6). Nowadays, HBV vaccines, routinely the second generation, are administered three times (on 0,1 and 6 months period) by intramuscular (deltoid) injection. Anti-HBs antibody concentration above $10 \mathrm{IU} / \mathrm{L}$ is considered efficient. In cases with concentration below 10 IU/L like decreasing the concentration with time, immunosuppression, smoking, obesity, renal failure and liver disease a booster dose or a revaccination are suggested $(1,5)$.

\section{Special Groups Needs Special Attention}

The effectiveness of HBV vaccination and its response are studied in some particular diseases and conditions like patients with diabetes mellitus (DM), HIV, inflammatory bowel disease, chronic kidney disease, end stage renal disease requiring hemodialysis and the newborn infants (4, 7-10). However, we still lack data in some groups, as an instance, our data about is not still efficient about the effectiveness of vaccination during pregnancy on the prevalence of newborn infection and its side effects (11).

\subsection{Diabetes Mellitus}

Schillie et al. concluded that adult patients with DM have a reduced response to HBV vaccination and suggested an extended interval between the final doses of vaccine or using additional series of vaccination in this group of patients (8). 


\subsection{Patients With HIV Infection}

HBV vaccination response is also reduced by HIV infection and some methods are available for improving this response like: using additional doses or adjuvants and intradermal injection. It is also believed that control of HIV replication and using of active antiviral therapy is of use for increasing the HBV vaccination response (10).

\subsection{Patients Who Undergo Hemodialysis}

Evidences show that patients who undergo HD cannot always produce enough antibodies in response to HBsAg following HBV vaccination; hence, infection with HBV remains a major health problem in these patients, with a high rate of morbidity and mortality (12-16). Although the first generation of HBV vaccine can protect these patients against the infection, it is less effective in them than in the normal population. In addition, it is reported that second generation of HBV vaccine has the same effect of the first generation in healthy people (1).

Some factors seem to be responsible for suboptimal response of HBV vaccination in patients who undergo HD; for example HLA-A1, B8, DR3 have been detected to have a higher prevalence in patients with lower vaccination responses (17). Female patients who undergo HD are more likely to be response to HBV vaccination than the male ones (17). Older age, malnutrition and infection with hepatitis $C$ virus and human immunodeficiency virus can also have some effects on reduction of the effectiveness of $\mathrm{HBV}$ vaccination (18-21). Anemia is a common finding among patients who undergo HD which is usually corrected by human recombinant erythropoietin (EPO). In a series of 97 patients, resistance to EPO was associated with lower anti-HBs levels and seroconversion (22). It seems to be partly due to poor nutrition status and inflammation in these individuals (22). However, Fabrizi and his colleagues found that EPO injection has no effects on the HBV vaccination in patients who undergo $\mathrm{HD}$ (14).

It has been reported that several methods can improve the effect of $\mathrm{HBV}$ vaccination and its response in these patients. Fabrizi et al. showed that using of higher thymopentin doses as an adjuvant can lead to an increase in seroresponse rate of HBV vaccination (13). Some other adjuvants were also investigated. It seems that levamisole can also be effective for increasing the HBV vaccine response in the HD patients (15), but for a better decision more studies in this field are essential. HBV-ASO4 containing adjuvant 3-O-desacyl-40-monophosphoryl lipid A which compatible with Engerix B Standard, makes an earlier and greater response to the $\mathrm{HBV}$ vaccination compared to the routine vaccination (1). Some studies have also evaluated the effect of intradermal and intramuscular injection of HBV vaccination in patients who undergo HD. It is said that in a short time follow up, intradermal route of HBV vaccination can lead to a stronger response than the intramuscular route; however, long term follow up did not show a meaningful difference between these two ways of injection (23).

\section{The Present Situation Several Years After Starting the Successful HBV Vaccination Program}

As mentioned above, it is well known that the HBV vaccination can lead to reduction of HBV prevalence and its complications, but now after many years of starting the HBV vaccination program there are still things to be taken care of. In a cross-sectional study by Al Ghamdi et al. evaluated the efficacy of primary HBV immunization in 238 medical students after 14-24 years (24). They found that their anti-HBs levels were meaningfully low (24). It has been said that with a worldwide immunization against $\mathrm{HBV}$, some mutations like HBV's protein mutation (25) may occur in the HBV genome and this can be lead to higher levels of failure in making an appropriate response to the HBV vaccination. Chiara and coworkers showed that even a booster dose of HBV vaccination could not increase the anti-HBs antibody levels more than $10 \mathrm{IU} / \mathrm{L}$ in individuals with decreased response of HBV vaccination (anti-HBs antibody less than $2 \mathrm{IU} / \mathrm{L}$ ) (26). In an study in Egypt It has also been reported that children with diabetics may not have effective concentrations of anti-HBs antibody after the primary vaccination (27). Moreover, there is a report of low long-term humoral immunity against HBV infection in vaccinated people in Bolivia (28). Patients with HIV infection may also lose their HBV vaccination response rapidly after completing the vaccination, so they need to be evaluated 6-12 months after finishing the primary vaccination series (29). Subjects with Down syndrome are another group that need a special follow up after $\mathrm{HBV}$ vaccination because of their low rate of seroconversion in response to the HBV vaccination (30). Additionally, patients with liver cirrhosis may also have a lower response to the HBV vaccination compared to the healthy population (31). Furthermore, many healthcare personnel may experience continuous lack of immunity to hepatitis B after completing vaccine series (32). Yildiz and colleagues evaluated the effect of HBV vaccine among children with steroid sensitive nephrotic syndrome and they observed a low response to the HBV vaccination and some relapses due to this vaccination in these cases. Finally, they suggested that the HBV vaccination should only be used in patients on low dose or withdrawal of steroid therapy (33).

Some studies suggested that using of a third generation vaccination in non- or low responder individuals to conventional vaccination can lead to making an effective level of anti-HBs antibody (more than $10 \mathrm{IU} / \mathrm{l}$ ) (34); moreover, adding adjuvants like Advax to the current vaccines may be helpful for improvement of immunity level in low responder individuals (35). Also HEPLISAV, as a HBV vaccine, is suggested as a good option to be used among different groups such as healthcare personnel, patients 
with chronic kidney disease and healthy population. It can give an earlier seroprotection with fewer times and doses compared with Engerix-B as a second generation HBV vaccine $(36,37)$.

Despite the advances in HBV vaccine development and prevention therapy, we are now faced with following questions. When the booster dose and in which groups it must be administered? How exactly we should act with the time decreasing response to the HBV vaccination? Does HBV genome mutations should be considered for current HBV vaccination program? Is adding new adjuvants mentioned in databases, necessary? Which adjuvants and also which generations are better for each special group? To get a better understanding about these points, both original and secondary studies seem to be essential.

\section{Acknowledgments}

Authors have nothing to declare.

\section{Authors' Contribution}

Both authors worked equally.

\section{Financial Disclosure}

Authors declare there is no conflict of interest.

\section{Funding/Support}

Authors have nothing to declare.

\section{References}

1. Edey M, Barraclough K, Johnson DW. Review article: Hepatitis B and dialysis. Nephrology (Carlton). 2010;15(2):137-45.

2. Liaw YF. Natural history of chronic hepatitis B virus infection and long-term outcome under treatment. Liver Int. 2009;29 Suppl 1:100-7.

3. World health organization (WHO). Global alert and response. Hepatitis B. Available from: http://www.who.int/csr/disease/hepatitis/ whocdscsrlyo20022/en/index2.html.

4. Grzegorzewska AE. Hepatitis B vaccination in chronic kidney disease: review of evidence in non-dialyzed patients. Hepat Mon. 2012;12(11).

5. Aspinall EJ, Hawkins G, Fraser A, Hutchinson SJ, Goldberg D. Hepatitis B prevention, diagnosis, treatment and care: a review. Occup Med (Lond). 2011;61(8):531-40.

6. Luo Z, Li L, Ruan B. Impact of the implementation of a vaccination strategy on hepatitis B virus infections in China over a 20year period. Int J Infect Dis. 2012;16(2):e82-8.

7. Schillie SF, Murphy TV. Seroprotection after recombinant hepatitis B vaccination among newborn infants: a review. Vaccine. 2013;31(21):2506-16.

8. Schillie SF, Spradling PR, Murphy TV. Immune response of hepatitis B vaccine among persons with diabetes: a systematic review of the literature. Diabetes Care. 2012;35(12):2690-7.

9. Sempere L, Almenta I, Barrenengoa J, Gutierrez A, Villanueva CO de-Madaria E, et al. Factors predicting response to hepatitis B vaccination in patients with inflammatory bowel disease. Vaccine. 2013;31(30):3065-71.

10. Whitaker JA, Rouphael NG, Edupuganti S, Lai L, Mulligan MJ. Strategies to increase responsiveness to hepatitis B vaccination in adults with HIV-1. Lancet Infect Dis. 2012;12(12):966-76.

11. Sangkomkamhang US, Lumbiganon P, Laopaiboon M. Hepatitis
$B$ vaccination during pregnancy for preventing infant infection. Cochrane Database Syst Rev. 2011;(3):CD007879.

12. Einollahi B. Immune response to hepatitis B vaccine in patients with chronic kidney disease. Hepat Mon. 2011;11(10):781-2.

13. Fabrizi F, Dixit V, Martin P. Meta-analysis: the adjuvant role of thymopentin on immunological response to hepatitis $\mathrm{B}$ virus vaccine in end-stage renal disease. Aliment Pharmacol Ther. 2006;23(11):1559-66.

14. Fabrizi F, Dixit V, Martin P, Messa P. Erythropoietin use and immunogenicity of hepatitis B virus vaccine in chronic kidney disease patients: a meta-analysis. Kidney Blood Press Res. 2012;35(6):50410.

15. Sanadgol H. Levamisole usage as an adjuvant to hepatitis B vaccine in hemodialysis patients, yes or no? Nephrourol Mon. 2013;5(1):673-8.

16. Yassin MH, Gupta V. Role of infection control in prevention of hepatitis B virus (HBV) in hemodialysis (HD) patients. Infect Disord Drug Targets. 2013;13(3):162-8.

17. Pol S, Legendre C, Mattlinger B, Berthelot P, Kreis H. Genetic basis of nonresponse to hepatitis B vaccine in hemodialyzed patients. J Hepatol.1990;11(3):385-7.

18. Ahuja TS, Kumar S, Mansoury H, Rodriguez H, Kuo YF. Hepatitis B vaccination in human immunodeficiency virus-infected adults receiving hemodialysis. Kidney Int. 2005;67(3):1136-41.

19. Fabrizi F, Martin P, Dixit V, Bunnapradist S, Dulai G. Meta-analysis: the effect of age on immunological response to hepatitis $B$ vaccine in end-stage renal disease. Aliment Pharmacol Ther. 2004;20(10):1053-62.

20. Fernandez E, Betriu MA, Gomez R, Montoliu J. Response to the hepatitis $B$ virus vaccine in haemodialysis patients: influence of malnutrition and its importance as a risk factor for morbidity and mortality. Nephrol Dial Transplant.1996;11(8):1559-63.

21. Navarro JF, Teruel JL, Mateos ML, Marcen R, Ortuno J. Antibody level after hepatitis B vaccination in hemodialysis patients: influence of hepatitis C virus infection. Am J Nephrol.1996;16(2):95-7.

22. Afsar B. The relationship between erythropoietin resistance and antibody response to hepatitis $\mathrm{B}$ vaccine in hemodialysis patients. Nephrourol Mon. 2013;5(3):806-12.

23. Fabrizi F, Dixit V, Magnini M, Elli A, Martin P. Meta-analysis: intradermal vs. intramuscular vaccination against hepatitis B virus in patients with chronic kidney disease. Aliment Pharmacol Ther. 2006;24(3):497-506.

24. Al Ghamdi SS, Fallatah HI, Fetyani DM, Al-Mughales JA, Gelaidan AT. Long-term efficacy of the hepatitis B vaccine in a high-risk group. J Med Virol. 2013;85(9):1518-22.

25. Bian T, Yan H, Shen L, Wang F, Zhang S, Cao Y, et al. Change in hepatitis $B$ virus large surface antigen variant prevalence 13 years after implementation of a universal vaccination program in China. J Virol. 2013;87(22):12196-206.

26. Chiara F, Bartolucci GB, Cattai M, Piazza A, Nicolli A, Buja A, et al Hepatitis B vaccination of adolescents: Significance of non-protective antibodies. Vaccine. 2013;32(1):62-8.

27. Elrashidy H, Elbahrawy A, El-Didamony G, Mostafa M, George NM, Elwassief A, et al. Antibody levels against hepatitis B virus after hepatitis B vaccination in Egyptian diabetic children and adolescents. Hum Vaccin Immunother. 2013;9(9):2002-6.

28. Masuet-Aumatell C, Ramon-Torrell JM, Casanova-Rituerto A Banque-Navarro M, Davalos-Gamboa Mdel R, Rodriguez SL. Seroprevalence of hepatitis B in two period birth cohorts of Bolivian children: effect of universal vaccination. Trans R Soc Trop Med Hyg. 2013;107(9):578-83.

29. Mizusawa M, Perlman DC, Lucido D, Salomon N. Rapid loss of vaccine-acquired hepatitis B surface antibody after three doses of hepatitis B vaccination in HIV-infected persons. Int J STD AIDS. 2013.

30. Nisihara R, De Bem RS, Negreiros PH, Utiyama SR, Oliveira NP, Amarante H. Low hepatitis B vaccine response in children with Down syndrome from Brazil. Child Care Health Dev. 2013.

31. Roni DA, Pathapati RM, Kumar AS, Nihal L, Sridhar K, Tumkur Rajashekar S. Safety and efficacy of hepatitis B vaccination in cirrhosis of liver. Adv Virol. 2013;2013:196704.

32. Kaltsas A, Sepkowitz K. Vaccinations for healthcare personnel: 
update on influenza, hepatitis B, and pertussis. Curr Opin Infect Dis. 2013;26(4):366-77.

33. Yildiz N, Sever L, Kasapcopur O, Cullu F, Arisoy N, Caliskan S. Hepatitis B virus vaccination in children with steroid sensitive nephrotic syndrome: immunogenicity and safety? Vaccine. 2013;31(33):3309-12.

34. Rendi-Wagner P, Shouval D, Genton B, Lurie Y, Rumke H, Boland $\mathrm{G}$, et al. Comparative immunogenicity of a PreS/S hepatitis B vaccine in non- and low responders to conventional vaccine. Vaccine. 2006;24(15):2781-9.

35. Saade F, Honda-Okubo Y, Trec S, Petrovsky N. A novel hepatitis
B vaccine containing Advax, a polysaccharide adjuvant derived from delta inulin, induces robust humoral and cellular immunity with minimal reactogenicity in preclinical testing. Vaccine 2013;31(15):1999-2007.

36. Eng NF, Bhardwaj N, Mulligan R, Diaz-Mitoma F. The potential of 1018 ISS adjuvant in hepatitis B vaccines: HEPLISAV review. Hum Vaccin Immunother. 2013;9(8):1661-72.

37. Kuan RK, Janssen R, Heyward W, Bennett S, Nordyke R. Cost-effectiveness of hepatitis B vaccination using HEPLISAV in selected adult populations compared to Engerix-B(R) vaccine. Vaccine. 2013;31(37):4024-32. 